\title{
Verification of Array, Record, and Pointer Operations in Pascal
}

\author{
DAVID C. LUCKHAM \\ Artificial Intelligence Laboratory, Stanford University \\ and \\ NORIHISA SUZUKI \\ Xerox Palo Alto Research Center
}

\begin{abstract}
A practical method is presented for automating in a uniform way the verification of Pascal programs that operate on the standard Pascal data structures Array, Record, and Pointer. New assertion language primitives are introduced for describing computational effects of operations on these data structures. Axioms defining the semantics of the new primitives are given. Proof rules for standard Pascal operations on data structures are then defined using the extended assertion language. An axiomatic rule for the Pascal storage allocation operation, NEW, is also given. These rules have been implemented in the Stanford Pascal program verifier. Examples illustrating the verification of programs which operate on list structures implemented with pointers and records are discussed. These include programs with side effects.
\end{abstract}

Key Words and Phrases: program verification, data structures, formal semantics, axiomatic semantics, pointers, Pascal, side effect, storage allocation

CR Categories: $4.34,4.49,5.24$

\section{INTRODUCTION}

Axiomatic proof rules are presented for the Pascal operations on data structures of type Array, Record, and Pointer. These proof rules are defined in an extension of the Floyd-Hoare logic of programs. There are in fact exactly two rules: an axiom for assignment to a selected part of any Pascal complex data structure (i.e., a data structure definable by a set of Pascal definitions of types Array, Record, and Pointer), and an axiom for storage allocation. In the case of pointers, our axiomatic rules offer a simple alternative definition of the semantics of Pascal pointer operations to the previous studies in $[1,6,15,17]$. The simplicity and practicality of the rules has already been tested by implementing them in a verifier (i.e., a program for automatically constructing proofs of correctness of Pascal programs). This verifier was then used to obtain proofs of Pascal programs that operate on complex data structures, including the Schorr-Waite marking

Permission to copy without fee all or part of this material is granted provided that the copies are not made or distributed for direct commercial advantage, the ACM copyright notice and the title of the publication and its date appear, and notice is given that copying is by permission of the Association for Computing Machinery. To copy otherwise, or to republish, requires a fee and/or specific permission.

This work was supported in part by the Advanced Research Projects Agency, Department of Defense, under Contracts DAHC 15-73-C-0435 and FF44620-73-C-0074.

Authors' addresses: D.C. Luckham, Artificial Intelligence Laboratory, Stanford University, Stanford, CA 94305; N. Suzuki, Xerox Palo Alto Research Center, Palo Alto, CA 94304.

(C) 1979 ACM 0164-0925/79/1000-0226 $\$ 00.75$

ACM Transactions on Programming Languages and Systems, Vol. 1, No. 2, October 1979, Pages 226-244. 
algorithm for garbage collection [18], and a simple FIFO scheduler for implementing the monitor construct [9].

The standard Pascal operations on variables and terms of types Array, Record, and Pointer are

(i) simple assignment, e.g., $\mathrm{X}:=\mathrm{Y}$;

(ii) selection of an element, e.g., IF X $\uparrow[\mathrm{I}] \mathrm{THEN} \ldots$...;

(iii) assignment to a selected element, e.g., $\mathrm{X} \uparrow:=\mathrm{E} ; \mathrm{X} \uparrow . \mathrm{F}:=\mathrm{G} ; \mathrm{X} \uparrow . \mathrm{F}[\mathrm{I}]:=\mathrm{H}$;

(iv) dynamic storage allocation, e.g., $\mathrm{NEW}(\mathrm{X}[\mathrm{I}])$, where $\mathrm{X}[\mathrm{I}]$ is a pointer.

Such operations must obey the strict type compatibility requirements of the language. Type compatibility except for subrange types can be checked by a parser and is outside the scope of this paper.

In Section 2 we introduce an assertion language for data structures. Assertions in this language specify the effects of data structure operations. An important feature is the use of reference classes to make assertions about pointer operations. The new assertion language extends the assertion language of $[5,6,8]$ normally used to specify Pascal programs. Using this new assertion language we define a single proof rule for all Pascal operations in categories (i), (ii), and (iii), and a second equally simple rule for storage allocation. We give some hand proofs illustrating use of the rules. The rule for assignment is a generalization of previous proof rules for arrays [10,14]. We observe that this rule is the cause of combinatorial explosion of the size of verification conditions when it is applied to sequences of complex data structure assignments. We introduce the notion of selector sequences to solve the problem.

Section 3 deals with some of the problems involved in automating more complicated proofs using our rules, in particular the need for user-defined concepts describing the properties of complex structures. Our assertion language for data structures does not express high level properties such as the loopfreeness of list structures. We give examples of how the programmer can introduce auxiliary predicates to define high level concepts. He can then specify the operation of his programs using these auxiliary predicates and verify their correctness. We also show how bugs in complex data manipulations can sometimes be detected from unsuccessful verification attempts. Section 3 contains some facts about verifiers necessary for the discussion; the reader will find a general introduction in [12, 18] to the kind of verifiers we are considering here. Familiarity with specifying programs and proving properties of them in Floyd-Hoare logic is assumed [5].

\section{Notation and Conventions}

The standard notation from $[5,8]$ is used. In addition we emphasize the following:

1. The textual substitution of $\mathbf{E}$ for all free occurrences of $\mathrm{X}$ in $\mathrm{P}$ is denoted by $\mathrm{P} \mid \mathrm{X}$.

2. "Variable" means Pascal variable [21].

3. Verification condition is abbreviated to VC.

4. $\Rightarrow$ is logical implication; $\rightarrow$ is a transformation.

5. Program specifications are inductive assertions included with the code; there are different kinds, entry/exit assertions, loop invariants, etc. $[5,8,12,18]$.

6. Comments in program text appear between percent signs. 


\section{AXIOMATIC PROOF RULES FOR OPERATIONS ON COMPLEX DATA STRUCTURES}

We assume that programs to be verified are accompanied by assertions (or specifications) stating the intended meaning of the programs. The language for writing assertions is distinct from the programming language and is called the assertion language. It consists of Pascal Boolean expressions with the addition of quantifiers and arbitrary (user-defined) predicates $[8,18]$. This is the specification language used in most program verification studies $[1,2,3,5,6,8,10,19]$. Our first concern in this section is to define an extension of this assertion language to permit assertions describing complex data structures (Section 2.1). Using this extended assertion language, we then define axioms for Pascal operations on complex data structures (Section 2.2).

The earliest proof rules for assignment and selection operations on state vectors were given by McCarthy [14]. These rules may be restated for arrays. In Hoare's notation they are

$$
\mathrm{P}|\underset{\text { assign(A, I, E) }}{\mathrm{A}}\{\mathrm{A}[\mathrm{I}]:=\mathrm{E}\} \mathrm{P}, \quad \mathrm{P}| \underset{\operatorname{select}(\mathrm{A}, \mathrm{I})}{\mathrm{X}}\{\mathrm{X}:=\mathrm{A}[\mathrm{I}]\} \mathrm{P}
$$

where assign(A, I, E) and select(A, I) are special functions introduced into the first-order assertion language to express operations on arrays; they obey the axioms given in [14], e.g.,

$$
\operatorname{select}(\operatorname{assign}(A, I, E), J)=_{d f} \quad \text { if } I=J \text { then } E \text { else } \operatorname{select}(A, J) \text {. }
$$

The set of axioms given in [14] define a first-order assertion language for array operations. Using assertions about assign (A, I, E) and select(A, J) it is easy to give Floyd-Hoare style axioms defining Pascal array operations. It is also possible to make inductive assertions describing the computation states of programs that manipulate arrays. Proofs of correctness of such programs can then be given within the Floyd-Hoare logic [5]. Axiomatic rules such as (1) above are easy to implement, and in various notational forms they have been the basis for automating the verification of array programs $[8,10,19]$.

We propose simply to extend this assertion language for arrays to Pascal operations on records and pointers, and generally to Pascal operations on any complex structure that can be built out of arrays, records, and pointers by recursive type declarations. To do this we shall first introduce "assign" and "select" functions on records and pointers. Pointers present a slight conceptual problem since there is no name in Pascal for the structure that is being assigned into by an assignment to a dereferenced pointer, e.g., $\mathrm{X} \uparrow:=\mathrm{E}$. In the analogous case, $\mathrm{A}[\mathrm{I}]:=\mathrm{E}$, the identifier $\mathrm{A}$ names the structure which is manipulated by the operation. We introduce reference class names for this purpose. Similarly, we introduce a storage allocation function into the assertion language. This requires a special predicate, PointerTo(X, S) to express when pointer $\mathrm{X}$ is pointing to a member of the reference class $S$, which we also add to the assertion language.

Before we begin the formal definitions, a remark about notation is in order. The notation used below for assign, select, etc., is what we have been using in implementations. For example, we denote $\operatorname{assign}(\mathrm{A}, \mathrm{I}, \mathrm{E})$ by $\langle\mathrm{A},[\mathrm{I}], \mathrm{E}\rangle$, and select(A, I) by A[I]. This is adequate for defining our axioms. But in actual proofs involving lengthy sequences of array operations, the resulting terms are not only 
unreadable but also cause a combinatorial explosion in the length of array terms in assertions. A solution to these problems is discussed in Section 2.3.

\subsection{Assertion Language for Data Structures}

The ordinary first-order assertion language is extended to express the effects of data structure operations. The newly introduced functions are defined axiomatically.

2.1.1 Reference Class Identifiers. We introduce new individual variables called reference class identifiers into the assertion language. They have the form

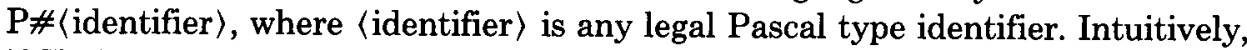
if T0 is declared as TYPE T0 $=\uparrow$ T (Pascal notation for "type Pointer to T"), then $\mathrm{P \# T}$ represents an unbounded set of data structures of type $\mathrm{T}$ that pointer variables of type T0 may refer to. Reference classes are not elements in Pascal (although the syntax for bounded reference classes appears in the early version of the Pascal language definition [21]). They are assertion language primitives and behave very much like unbounded arrays. We introduce reference class types in the assertion language. The type of $\mathrm{P \# T}$ is reference class of $\mathrm{T}$.

2.1.2 Functions and Predicates on Data Structures. We introduce function symbols corresponding to the Pascal selection, assignment, and memory allocation operations on complex data type variables:

selection: $\mathrm{X}[\mathrm{Y}]$ (array selection), R.F (record selection), DCQ (reference class selection).

assignment: $\langle\mathrm{X},[\mathrm{Y}], \mathrm{Z}\rangle$ (array assignment), $\langle\mathrm{R}, \mathrm{F}, \mathrm{Z}\rangle$ (record assignment), $(\mathrm{D}, \subset \mathrm{Q} \supset, \mathrm{Z}\rangle$ (reference class assignment).

extension: $\mathrm{D} \cup\{\mathrm{Q}\}$.

The definition of terms in the assertion language is extended to accommodate new terms created by the combination of terms, reference class identifiers, and the special functions. These new terms are called data structure terms and can be rigorously defined as follows:

1. all Pascal variables,

2. all terms obtained from 1 and the new functions by function composition.

The data structure terms must obey the Pascal type compatibility requirements. Thus $\mathrm{X}[\mathrm{Y}]$ is legal only if $\mathrm{X}$ is of array type and $\mathrm{Y}$ is the correct index type. Similarly, $\langle X, \subset Y \supset, Z\rangle$ is legal only if $X$ is of type reference class and $Y, Z$ have types compatible with $\mathrm{X}$. Types of data structure terms are determined according to the following rules. The type of $\mathrm{X}[\mathrm{Y}]$ is the type of elements of the array term $\mathrm{X}$. The type of $\langle\mathrm{X},[\mathrm{Y}], \mathrm{Z}\rangle$ is the same as that of $\mathrm{X}$. If the type of $\mathrm{X}$ is reference class of $T$, the type of $X \subset Y \supset$ is $T$. The type of $\langle X, \subset Y \supset, Z\rangle$ is the same as that of $\mathrm{X}$. The type of $\mathrm{D} \cup\{\mathrm{X}\}$ is the same (reference class) type as $\mathrm{D}$. The types for record terms are defined analogously.

A new predicate symbol is introduced to specify whether a pointer refers to an object in a reference class.

reference predicate: PointerTo(X,D) means $\mathrm{X}$ is a pointer which points to a member of the reference class $D$. 
PointerTo(X, D) also follows the type compatibility requirement: $\mathrm{D}$ is a reference class of type $T$ and $X$ is of type $\uparrow T$.

2.1.3 Axioms for Data Structure Terms. Data structure terms of types Array, Record, and Reference Class are equal if they have the same type and are component-wise equal; reference class terms are equal if in addition they have the same number of components. For example,

If $A, B$ are Arrays of type ARRAY $[m \ldots n]$ of $T, A=B \equiv(\forall i)(A[i]=B[i])$

If $\mathrm{C}, \mathrm{D}$ are Records of type RECORD sl: Tl; . . ; sn: Tn END, C $=\mathrm{D} \equiv \mathrm{C} . \mathrm{sl}=\mathrm{D} . \mathrm{sl} \wedge$ $\ldots \wedge$ C.sn $=$ D.sn.

If $\mathrm{E}, \mathrm{F}$ are Reference Classes of type Reference Class of $\mathrm{T}, \mathrm{E}=\mathrm{F} \equiv(\forall \mathrm{x})$ (PointerTo $(\mathrm{x}, \mathrm{E}) \equiv \operatorname{PointerTo}(\mathrm{x}, \mathrm{F})) \wedge(\forall \mathrm{x})(\operatorname{PointerTo}(\mathrm{x}, \mathrm{E}) \Rightarrow \mathrm{E} \subset \mathrm{x} \supset=\mathrm{F} \subset \mathrm{x} \supset)$.

The selection and assignment functions satisfy the following axioms (all the free variables are universally quantified):

$$
\begin{array}{ll}
\text { Axiom } & \text { 1. } \mathrm{Y}=\mathrm{U} \Rightarrow\langle\mathrm{X},[\mathrm{Y}], \mathrm{Z}\rangle[\mathrm{U}]=\mathrm{Z} \\
\text { Axiom } & \text { 2. } \mathrm{Y} \neq \mathrm{U} \Rightarrow\langle\mathrm{X},[\mathrm{Y}], \mathrm{Z}\rangle[\mathrm{U}]=\mathrm{X}[\mathrm{U}] \\
\text { Axiom } & \text { 3. }\langle\mathrm{X}, \mathrm{Y}, \mathrm{Z}\rangle . \mathrm{Y}=\mathrm{Z} \\
\text { Axiom } & \text { 4. }\langle\mathrm{X}, \mathrm{Y}, \mathrm{Z}\rangle \mathrm{U}=\mathrm{X} . \mathrm{U}, \text { where } \mathrm{Y} \text { and } \mathrm{U} \text { are distinct identifiers } \\
\text { Axiom } & \text { 5. } \mathrm{Y}=\mathrm{U} \Rightarrow\langle\mathrm{X}, \subset \mathrm{Y} \supset, \mathrm{Z}\rangle \subset \mathrm{U} \supset=\mathrm{Z} \\
\text { Axiom } & \text { 6. } \mathrm{Y} \neq \mathrm{U} \Rightarrow\langle\mathrm{X}, \subset \mathrm{Y} \supset, \mathrm{Z}\rangle \subset \mathrm{U} \supset=\mathrm{X} \subset \mathrm{U} \supset
\end{array}
$$

The extension function obeys three axioms:

$$
\begin{aligned}
& \text { Axiom 7. } \mathrm{D} \cup\{\mathrm{X}\} \cup\{\mathrm{Y}\}=\mathrm{D} \cup\{\mathrm{Y}\} \cup\{\mathrm{X}\} \\
& \text { Axiom 8. } \mathrm{X} \neq \mathrm{Y} \Rightarrow(\mathrm{D} \cup\{\mathrm{X}\}) \subset \mathrm{Y} \supset=\mathrm{D} \subset \mathrm{Y} \supset \\
& \text { Axiom 9. } \mathrm{X} \neq \mathrm{Y} \Rightarrow\langle\mathrm{D}, \subset \mathrm{Y} \supset, \mathrm{Z}\rangle \cup\{\mathrm{X}\}=\langle\mathrm{D} \cup\{\mathrm{X}\}, \subset \mathrm{Y} \supset, \mathrm{Z}\rangle
\end{aligned}
$$

The predicate PointerTo(X, D) obeys the following axioms:

Axiom 10. PointerTo(NIL, D)

Axiom 11. PointerTo(X, D $\cup\{\mathrm{X}\})$

Axiom 12. PointerTo(X, $\langle\mathrm{D}, \subset \mathrm{Y} \supset, \mathrm{E}\rangle) \equiv \operatorname{PointerTo}(\mathrm{X}, \mathrm{D})$

Axiom 13. $\mathrm{X} \neq \mathrm{Y} \Rightarrow(\operatorname{PointerTo}(\mathrm{X}, \mathrm{D} \cup\{\mathrm{Y}\}) \equiv \operatorname{PointerTo}(\mathrm{X}, \mathrm{D}))$

Axiom 14. $(\forall \mathrm{D}(\exists \mathrm{X}) \neg$ PointerTo $(\mathrm{X}, \mathrm{D})$

Other standard lemmas may be derived from these axioms.

For example, $\langle\mathrm{A},[\mathrm{I}], \mathrm{A}[\mathrm{I}]\rangle=\mathrm{A}$ can be obtained in the following way:

From the equality axiom for array terms,

$$
\langle A,[I], A[I]\rangle=A \text { if and only if }(\forall j)(\langle A,[I], A[I]\rangle[j]=A[j]) .
$$

We prove the latter formula by cases.

Suppose $j \neq I$. Then, $\langle A,[I], A[I]\langle[j]=A[j]$ from axiom 2 .

Suppose $\mathrm{j}=\mathrm{I}$. Then, $\langle A,[\mathrm{I}], \mathrm{A}[\mathrm{I}]\rangle[\mathrm{j}]=\mathrm{A}[\mathrm{I}]=\mathrm{A}[\mathrm{j}]$ from axiom 1 .

In both cases $\langle A,[I], A[I]\rangle[j]=A[j]$. Therefore, $(\forall j)(\langle A,[I], A[I]\rangle[j]=A[j])$.

The assertion language for data structures consists of assertions that can be made using the new terms and the equality and PointerTo predicates. The theorems of this language (i.e., assertions provable from those axioms above) describe properties of data structures. 
Example. We can show that the assertion,

$$
(\mathrm{K} \neq \mathrm{I} \wedge \mathrm{L}=\mathrm{J}) \Rightarrow\langle\langle\mathrm{A},[\mathrm{I}],\langle\mathrm{A}[\mathrm{I}],[\mathrm{J}], 2\rangle\rangle,[\mathrm{K}], \mathrm{B}\rangle[\mathrm{I}][\mathrm{L}]=2
$$

is a theorem of this theory. By axiom $2, \mathrm{~K} \neq \mathrm{I} \Rightarrow\langle\langle\mathrm{A},[\mathrm{I}],\langle\mathrm{A}[\mathrm{I}],[\mathrm{J}], 2\rangle\rangle,[\mathrm{K}], \mathrm{B}\rangle$ $[\mathrm{I}][\mathrm{L}]=\langle\mathrm{A},[\mathrm{I}],\langle\mathrm{A}[\mathrm{I}],[\mathrm{J}], 2\rangle\rangle[\mathrm{I}][\mathrm{L}]$. Axiom 1 implies $\langle\mathrm{A},[\mathrm{I}],\langle\mathrm{A}[\mathrm{I}],[\mathrm{J}], 2\rangle\rangle[\mathrm{I}][\mathrm{L}]$ $=\langle\mathrm{A}[\mathrm{I}],[\mathrm{J}], 2\rangle[\mathrm{L}]$, and finally $\mathrm{L}=\mathrm{J} \Rightarrow\langle\mathrm{A}[\mathrm{I}],[\mathrm{J}], 2\rangle[\mathrm{L}]=2$.

Remark. Proofs similar to the above example can be found easily by automatic theorem provers. Therefore, if the correctness of a program can be reduced to proving assertions about data structures, then the proof of correctness can often be fully automated.

2.1.4 High Level Concepts. The assertion language for data structures expresses only the effects of the basic Pascal operations. In order to express many complicated properties of data structures we need to introduce auxiliary predicates. For example, if we have Pascal type definitions,

$$
\begin{aligned}
\text { type } \mathrm{T} 0 & =\uparrow \mathrm{T}, \\
\mathrm{T} & =\text { record } \ldots ; \text { Next: } \mathrm{T} 0 ; \ldots
\end{aligned}
$$

it may be necessary to make assertions about "reachability" between pointers, i.e., from pointer $x$ one can reach pointer $y$ by performing the Next operation finitely many times. We introduce auxiliary predicates and add the axioms (D ranges over terms of type reference class of $\mathrm{T})$ :

$$
\begin{aligned}
& \operatorname{Reach}(D, x, y) \equiv(\exists j) \operatorname{Reachstep}(D, x, y, j) \\
& \operatorname{Reachstep}(D, x, y, 0) \equiv(x=y) \\
& \text { Reachstep }(D, x, y, j+1) \equiv(\exists z) \operatorname{Reachstep}(D, x, z, j) \wedge D C z \supset . N e x t=y
\end{aligned}
$$

In Section 3 we present an alternative axiomatization of this property and its use in mechanical verification.

\subsection{Floyd-Hoare Style Axioms}

We introduce a convention for denoting dereferenced pointers in assertions, and two axiomatic proof rules for Pascal complex data structure operations.

2.2.1 Proof Rules. Assertion language terms may contain either $\mathrm{X} \uparrow$ or $\mathrm{P \# T} \subset \mathrm{X} \supset$, both of which denote the same object. We have permitted $\mathrm{X} \uparrow$ for the convenience of the user. The axioms (and their implementation in the verifier)

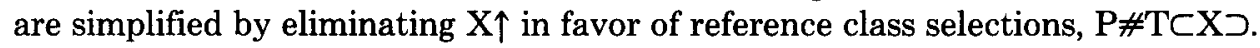

Elimination of Pascal dereference operations. In assertions, all dereferenced

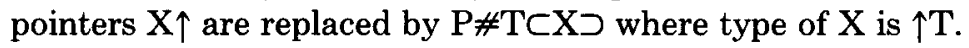

Examples. In the following examples we assume that type of $\mathrm{X}$ is $\uparrow \mathrm{T}$ and type of $\mathrm{X} \uparrow . \mathrm{F}$ is $\uparrow \mathrm{S}$. The left-hand side terms are transformed to right-hand side terms.

$$
\begin{aligned}
& \mathrm{X} \uparrow \rightarrow \mathrm{P \# T \subset X \supset} \\
& \mathrm{X} \uparrow . \mathrm{F} \rightarrow \mathrm{P \# T \subset X \supset . \textrm {F }} \\
& \mathrm{A}[\mathrm{X} \uparrow . \mathrm{F}] \rightarrow \mathrm{A}[\mathrm{P \# T \subset X \supset . \textrm {F } ]}
\end{aligned}
$$

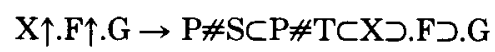

Note that the replacement must take place from inside out when there is a sequence of dereferencing $(\uparrow)$. 
This replacement can be formally defined using a transformation function $\operatorname{Tr}(\mathrm{e})$ on data structure terms e:

$$
\begin{aligned}
& \operatorname{Tr}(V)=V, \quad \text { if } V \text { is an identifier; } \\
& \operatorname{Tr}(\mathrm{Z} \uparrow)=\mathrm{P \#} \operatorname{T} \subset \operatorname{Tr}(\mathrm{Z}) \supset, \quad \text { where type of } \mathrm{Z} \text { is } \uparrow T \text {; } \\
& \operatorname{Tr}(\mathrm{A}[\mathrm{I}])=\operatorname{Tr}(\mathrm{A})[\operatorname{Tr}(\mathrm{I})] ; \\
& \operatorname{Tr}(\mathrm{R} . \mathrm{F})=\operatorname{Tr}(\mathrm{R}) . \mathrm{F} .
\end{aligned}
$$

In the following we assume that all dereferenced pointers have been eliminated from all assertions.

Rule 1 (Axiom Scheme for Assignment to any Pascal Variable).

$$
\text { Subst(E, V, Q) }\{\mathrm{V}:=\mathrm{E}\} \mathrm{Q}
$$

where $\mathrm{V}$ is a variable, $\mathrm{E}$ is an expression, $\mathrm{Q}$ is an assertion, and Subst is defined recursively on assertion language terms $\mathrm{V}$ by

$$
\begin{aligned}
& \operatorname{Subst}(E, V, Q)=\left.Q\right|_{E} ^{V}, \quad \text { if } V \text { is an identifier; } \\
& \operatorname{Subst}(E, A[I], Q)=\operatorname{Subst}((A,[I], E), A, Q) \text {; } \\
& \operatorname{Subst}(E, R . F, Q)=\operatorname{Subst}((R, . F, E\rangle, R, Q) \text {; }
\end{aligned}
$$

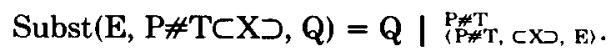

Example.

$$
\begin{aligned}
& \text { type } \quad \mathrm{P}=\uparrow \mathrm{T} ; \\
& \mathrm{T}=\operatorname{array}[1 \ldots N] \text { of integer; } \\
& \operatorname{var} \mathrm{X}: \mathrm{P} ;
\end{aligned}
$$

If we are given the declaration as above, the axiom for $\mathrm{X} \uparrow[\mathrm{I}]:=\mathrm{E}$ is

$$
\operatorname{Subst}(\mathrm{E}, \mathrm{P \# T} \subset \mathrm{X} \supset[\mathrm{I}], \mathrm{Q})\{\mathrm{X} \uparrow[\mathrm{I}]:=\mathrm{E}\} \mathrm{Q} \text {. }
$$

The steps in computing the Subst term are

$\operatorname{Subst}(E, P \# T \subset X \supset[I], Q)=$

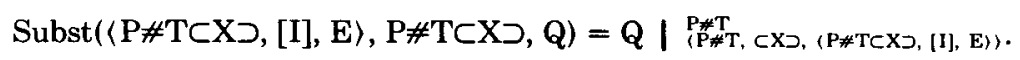

Rule 2 (Axiom Scheme for Storage Allocation).

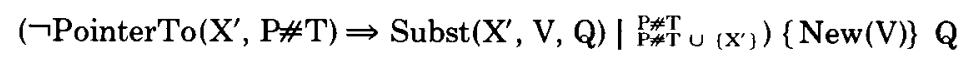

where type of $\mathrm{V}$ is $\uparrow T$, and $\mathrm{X}^{\prime}$ is a fresh identifier which does not appear anywhere.

The motivation for the predicate PointerTo is to give a complete rule for memory allocation. PointerTo(X, D) is introduced in assertions in order to express in rule 2 that the value of $\mathrm{X}$ after a $\mathrm{NEW}(\mathrm{X})$ is distinct from any pointer value existing previously. This is expressed by $\neg$ PointerTo $\left(\mathrm{X}^{\prime}, \mathrm{D}\right)$ in the precondition. Simple statements that are clearly intended to be true in Pascal like

$$
\operatorname{TRUE}\{\mathrm{New}(\mathrm{V})\} \mathrm{V} \neq \mathrm{NIL}
$$

and

$$
\text { PointerTo(X, P\#T) }\{\text { New(V) }\} \text { V } \neq \text { X }
$$


are easily proved using rule 2 and the axioms for PointerTo (axioms 10-13, Section 2.1.3). It can also be proved that operations on a new memory location do not change values in old locations.

Example.

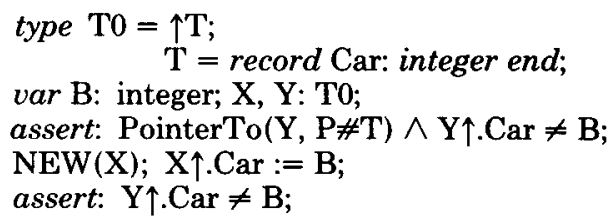

This program fragment is consistent with the assertions because the value of $X$ after $\mathrm{NEW}(\mathrm{X})$ is distinct from any previous pointer value, e.g., Y. A correctness proof can be given using our rules as follows:

1. The formula

$\langle\mathrm{P \# T}, \subset \mathrm{X} \supset,\langle\mathrm{P \# T \subset X \supset ,Car,B}\rangle) \subset \mathrm{Y} \supset . \mathrm{Car} \neq \mathrm{B}\{\mathrm{X} \uparrow . \mathrm{Car}:=\mathrm{B}\} \mathrm{P \# T \subset Y \supset .Car \neq}$ B

is an instance of rule 1 . Hence we try to prove

PointerTo(Y,P\#T) $\wedge \mathrm{P \# T \subset Y \supset .Car \neq B}$

$\{\mathrm{NEW}(\mathrm{X})\}\langle\mathrm{P \# T}, \subset \mathrm{X} \supset,\langle\mathrm{P \# T \subset X \supset ,.Car,} \mathrm{B}\rangle\rangle \subset \mathrm{Y} \supset . \mathrm{Car} \neq \mathrm{B}$.

By rule 2, this reduces to proving the following:

2. (PointerTo(Y, P\#T) $\wedge \mathrm{P \# T \subset Y \supset .Car} \neq \mathrm{B} \wedge \neg$ PointerTo $\left.\left(\mathrm{X}^{\prime}, \mathrm{P \# T}\right)\right) \Rightarrow$ $\left\langle\mathrm{P \# T} \cup\left\{\mathrm{X}^{\prime}\right\}, \subset \mathrm{X}^{\prime} \supset,\left\langle\mathrm{P \# T} \cup\left\{\mathrm{X}^{\prime}\right\} \subset \mathrm{X}^{\prime} \supset\right.\right.$, .Car, $\left.\left.\mathrm{B}\right\rangle\right\rangle \subset \mathrm{Y} \supset . \mathrm{Car} \neq \mathrm{B}$.

The premises imply $\mathrm{X}^{\prime} \neq \mathrm{Y}$. Using axioms 5,6 , and 8 , the conclusion is reduced to $\mathrm{P \# T \subset Y \supset .Car} \neq \mathrm{B}$, which is one of the premises. Therefore, we proved that the program is correct with respect to assertions.

The soundness and completeness of rules 1 and 2 relative to a model of computation on data structures are proved in [13].

2.2.2 Extensions to Pascal Axiomatic Semantics. We propose the following changes to the axiomatic semantics of Pascal given in [6]: (i) The assertion language $[6,8]$ is extended by adding assertions about data structure terms. (ii) The assignment axioms (11.1(1)-(4) of [6]) are replaced by rule 1. Assignment to a dereferenced pointer is now explicitly characterized by an axiom expressing a change to the associated reference class; in [6] its meaning is not defined. (iii) Rule 2 is introduced to define the semantics of the NEW operation; this replaces the description given in [6, p. 343 and 11.7, p. 346] in terms of variables $\xi$ and $\tau$ that "are not available to the Pascal programmer." (iv) Finally, changes are made to the other rules in [6] to express the semantics that wherever a dereferenced pointer variable appears in a program statement, the corresponding reference class is a global variable of that statement. For example, the procedure call rule in $[6]$ is

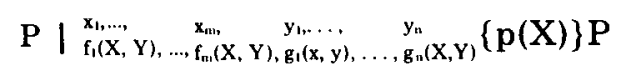

where $\mathrm{X}$ is the list of actual parameters, $\mathrm{x}_{1}, \ldots, \mathrm{x}_{\mathrm{m}}$ are those members of $\mathrm{X}$ corresponding to formal VAR parameters, $\mathrm{Y}$ is the list of all global variables of procedure $p$, and $y_{1}, \ldots, y_{n}$ are those members of $Y$ which are subject to assignments in $p$. There must be no aliasing between variables $x_{1}, \ldots, x_{m}$, 
$\mathrm{y}_{1}, \ldots, \mathrm{y}_{\mathrm{n}}$. The rule expresses procedure call as a simultaneous assignment $\mathrm{x}_{1}:=$ $f_{1}(X, Y), \ldots, y_{n}:=g_{n}(X, Y)$, where the $f_{1}$ and $g_{1}$ may be specified in the declaration of $p$. This rule must be changed as follows: if any dereferenced pointer of type $\uparrow T$ is subject to assignment by $p$, then P\#T is a member of $y_{1}, \ldots, y_{n}$. Reference classes are not legal variables in Pascal and cannot be named as parameters in procedure declarations. So the requirement that they be named as global variables is implemented in the verifier by introducing an explicit GLOBAL assertion in which all global variables of a procedure, including reference classes, must be named. Global variables are treated exactly as parameters in the procedure call rule.

Example.

$$
\begin{aligned}
& \text { procedure } \mathrm{F} 00 \text { ( }(\text { formal parameter list }\rangle) \text {; } \\
& \text { global (var } \mathrm{P \# T);}
\end{aligned}
$$

\subsection{Notational Considerations}

The notation we have presented simplifies the definition of data structure terms and the axioms 1-14. However, our experience shows that it causes a serious implementation problem. The size of verification conditions tends to grow at a rate exponential in the number of data structure assignments. We show such a phenomenon by an example.

Example. If the statement we want to prove is of the form

$$
\mathrm{R}\{\mathrm{X} \uparrow . \mathrm{Car}:=\mathrm{Y} ; \mathrm{X} \uparrow . \mathrm{Cdr}:=\mathrm{Z}\} \mathrm{Y} \uparrow . \mathrm{Car}=\mathrm{W}
$$

then the use of the assignment rule creates the following statement to prove:

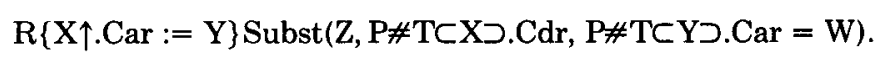

The expansion of the postcondition takes three steps:

$$
\begin{aligned}
& \text { Subst ( }(P \# T \subset X \supset, . C d r, Z\rangle, P \# T \subset X \supset, P \# T \subset Y \supset . C a r=W)
\end{aligned}
$$

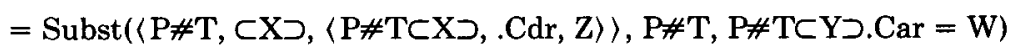

$$
\begin{aligned}
& =\langle\mathrm{P \# T}, \subset \mathrm{X} \supset,\langle\mathrm{P \# T} \subset \mathrm{X} \supset, \mathrm{Cdr}, \mathrm{Z}\rangle\rangle \subset \mathrm{Y} \supset . \mathrm{Car}=\mathrm{W} \text {. }
\end{aligned}
$$

Now there are two instances of $\mathrm{P \# T}$. The final verification condition created by one more use of the assignment rule has four instances of $\mathrm{P \# T}$ as shown here:

$$
\begin{aligned}
& \mathrm{R} \Rightarrow\langle\langle\mathrm{P} \# \mathrm{~T}, \subset \mathrm{X} \supset,\langle\mathrm{P} \# \mathrm{~T} \subset \mathrm{X} \supset, \mathrm{Car}, \mathrm{Y}\rangle\rangle, \subset \mathrm{X} \supset \text {, }
\end{aligned}
$$

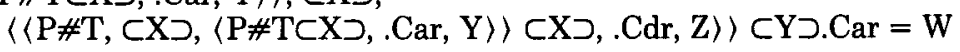

2.3.1 Selector Sequences. One cause of the explosion in the previous example is the multiple selectors in the left-hand side of assignments. To alleviate this problem we introduce the notion of selector sequences.

As one can see from the verification condition, there are definite regularities in the formulas created from the assignment with multiple selectors. They have the form $\langle\mathrm{D}, \subset \mathrm{X} \supset,\langle\mathrm{D} \subset \mathrm{X} \supset, . \mathrm{F}, \mathrm{E}\rangle\rangle$. We abbreviate this as $\langle\mathrm{D}, \subset \mathrm{X} \supset . \mathrm{F}, \mathrm{E}\rangle$. Here the new notation $\subset X \supset . F$ is called a selector sequence. In general, the abbreviation rule is

$$
\begin{aligned}
& \langle\mathrm{D}, \subset \mathrm{X} \supset,\langle\mathrm{D} \subset \mathrm{X} \supset, \mathrm{S}, \mathrm{E})\rangle \rightarrow\langle\mathrm{D}, \mathrm{CX} \supset \otimes \mathrm{S}, \mathrm{E}\rangle \\
& \langle\mathrm{D},[\mathrm{X}],\langle\mathrm{D}[\mathrm{X}], \mathrm{S}, \mathrm{E}\rangle) \rightarrow\langle\mathrm{D},[\mathrm{X}] \otimes \mathrm{S}, \mathrm{E}\rangle \\
& \langle\mathrm{D}, \mathrm{X},\langle\mathrm{D} . \mathrm{X}, \mathrm{S}, \mathrm{E})\rangle \rightarrow\langle\mathrm{D}, \mathrm{X} \otimes \mathrm{S}, \mathrm{E}\rangle
\end{aligned}
$$


where $S$ is a sequence of selectors and $\otimes$ stands for string concatenation (it does not actually appear in formulas).

This reduces the number of identifiers for data structures appearing in the verification conditions and eliminates the combinatorial explosion produced from common examples such as the one shown above.

The verification condition for the above example using the selector sequence notation is

$$
\mathrm{R} \Rightarrow\langle(\mathrm{P \# T}, \subset \mathrm{X} \supset . \mathrm{Car}, \mathrm{Y}\rangle, \subset \mathrm{X} \supset . \mathrm{Cdr}, \mathrm{Z}\rangle \subset \mathrm{Y} \supset . \mathrm{Car}=\mathrm{W}
$$

and $\mathrm{P \# T}$ appears only once instead of four times.

Selector sequences are used in the implementation of the rule of assignment. This is achieved by permitting selector sequences to occur in data structure terms as indicated by the abbreviation rule above and changing the definition of Subst.

The axioms for data structure terms (axioms 1-6, 9, 12, Section 2.2) change accordingly; for example,

$$
\begin{aligned}
& \langle\mathrm{D},[\mathrm{I}] \otimes \mathrm{L}, \mathrm{E}\rangle[\mathrm{J}]=\quad \text { if } \mathrm{I}=\mathrm{J} \text { then }\langle\mathrm{D}[\mathrm{I}], \mathrm{L}, \mathrm{E}\rangle \text { else } \mathrm{D}[\mathrm{J}], \\
& \langle\mathrm{D}, \varphi, \mathrm{E}\rangle=\mathrm{E},
\end{aligned}
$$

where $\mathrm{L}$ is a possibly empty sequence of selectors and $\varphi$ is an empty sequence of selectors.

The definition of Subst is

$$
\begin{aligned}
& \operatorname{Subst}(E, V, Q)=Q \mid \stackrel{V}{V} \quad \text { if } V \text { is an identifier, } \\
& \operatorname{Subst}(E, A \otimes S, Q)=\left.Q\right|_{(A, S, E)} ^{A} \quad \text { where } A \text { is an identifier. }
\end{aligned}
$$

Therefore, the assignment rule for a particular assignment like $\mathrm{X} \uparrow . \mathrm{Car}:=\mathrm{Y}$ is

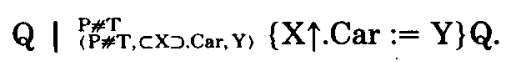

2.3.2 Names for Common Subterms. As we have demonstrated above, selector sequences can alleviate some of the combinatorial explosion problems caused by sequences of assignments. However, another cause is multiple occurrences of data structure identifiers (like $\mathrm{P \# T}$ ) in the user-supplied assertions. As we have shown in the previous example, the substitution term becomes quite large relative to the size of assertions. Therefore, multiple copies of the same terms cause another difficulty in readability as well as combinatorial explosion. A solution is to change the definition of $\operatorname{Subst}(E, X \otimes S, Q)$ again so that in the case where $E$ is a data structure term an equality is asserted instead of carrying out a textual substitution. The definition is

$$
\operatorname{Subst}(E, X \otimes S, Q)=_{\mathrm{df}}\langle\mathrm{X}, \mathrm{S}, \mathrm{E}\rangle=\mathrm{X}^{\prime} \Rightarrow \mathrm{Q} \mid \mathrm{X}_{\mathrm{X}^{\prime}}^{\mathrm{X}}
$$

where $\mathrm{X}$ is an identifier, $\mathrm{X}^{\prime}$ is a fresh identifier, and $\mathrm{S}$ is a selector sequence. This means that multiple occurrences of large data structure terms are never generated.

\section{EXAMPLES}

The extensions to the assertion language and proof rules defined in Section 2 have been implemented in the Stanford Pascal verifier. The verifier also uses axioms 1-13 (Section 2.1.3) to simplify VCs (verification conditions). Axiom 14 is required for logical completeness. It implies the existence of an unbounded set of pointer values; it is not normally required in verification. 


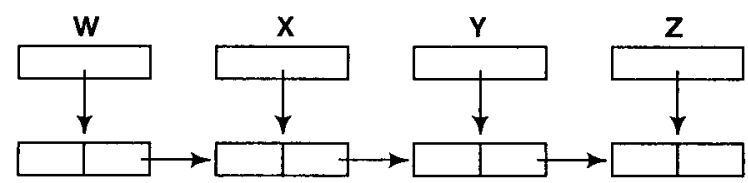

Fig. 1. State of reference class P\#Linear at L1

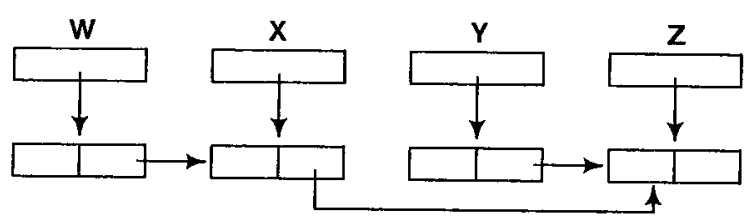

Fig. 2. State of reference class P\#Linear at L2

Some examples of specifying and verifying programs with pointer type parameters are given below. Details of the verifier and studies of other applications can be found in $[3,12,16,18,19]$.

\subsection{Side Effects in Pointer Data Structures}

\section{Example 1.}

type Linear $=$ record $\mathrm{Val}$ : integer; Next: Word end;

Word $=\uparrow$ Linear;

$\operatorname{var} \mathrm{W}, \mathrm{X}, \mathrm{Y}, \mathrm{Z}$ : Word;

begin

NEW(W); NEW(X); NEW(Y); NEW(Z);

$\mathrm{W} \uparrow$. Val $:=1$;

$\mathrm{W} \uparrow$. Next $:=\mathrm{X}$;

$\mathrm{X} \uparrow . \mathrm{Val}:=2$;

$\mathrm{X} \uparrow$. Next $:=\mathrm{Y}$;

$\mathrm{Y} \uparrow . \mathrm{Val}:=3$;

$\mathrm{Y} \uparrow$. Next : $=\mathrm{Z}$;

$\mathrm{Z} \uparrow . \mathrm{Val}:=4$;

$\mathrm{X} \uparrow$. Next $:=\mathrm{Z}$;

\% L1: At this point there is a four cell linear list

(Figure 1). \% assert $\mathrm{W} \uparrow$. Next $\uparrow$. Next $\uparrow$. Val $=4$ end.

$\% \mathrm{~L} 2$ : Now, $\mathrm{Y} \uparrow$ has been cut out of the linear list.

(Figure 2).\%

Figure 2 shows the final state of the reference class P\#Linear. The only operation involving $W \uparrow . N e x t \uparrow . N e x t \uparrow . V a l$ assigns 3 to the cell. That cell is then "short circuited" out of the list by an operation that does not explicitly mention it.

When this example is given to the verifier, the exit assertion is first transformed to $\mathrm{P \# Linear} \subset \mathrm{P} \#$ Linear $\subset \mathrm{P} \#$ Linear $\subset \mathrm{W} \supset$. Next $\supset . \mathrm{Next} \supset . \mathrm{Val}=4$. A verification condition is then constructed (below), and finally the result of attempting to automatically simplify and prove it is output (the user is not normally expected to try to analyze unsimplified conditions):

VERIFICATION CONDITION FOR THE MAIN PROGRAM IS:

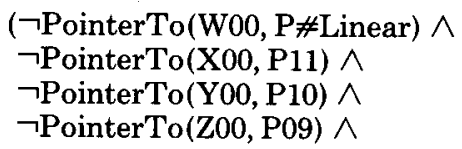




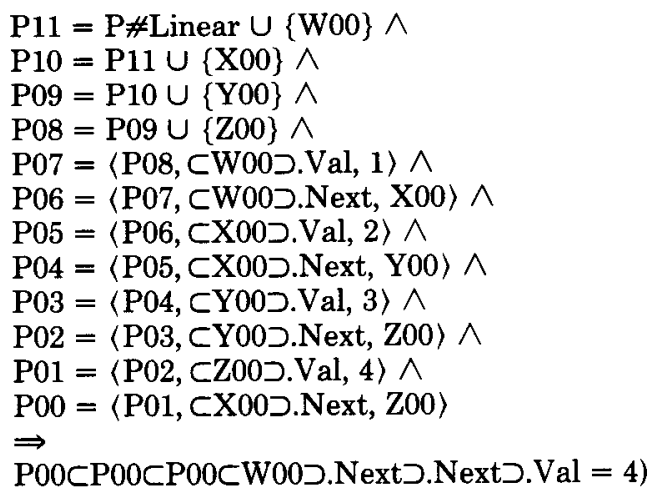

THE RESULT OF SIMPLIFICATION IS: TRUE

In this example the verifier automatically reduces the VC completely to TRUE with the reduction rules obtained from data structure axioms (axioms 5 and 6 , Section 2.1.3, and axioms for PointerTo) and no additional information is required from the user.

\subsection{Verification Bases}

Verifications normally depend on user-supplied lemmas. The reason for this is that many often used properties of complex data structures do not have a standard (i.e., universally accepted) axiomatization. The user can introduce auxiliary predicates in assertions to represent such properties. He must give lemmas defining the auxiliary predicates. The verifier uses these lemmas to simplify and prove VCs. If all VCs are reduced to TRUE this means that there is a proof that the program satisfies its specifications assuming the lemmas. The set of lemmas is called a basis of the verification. A basis is not necessarily a complete axiomatization of given programming concepts but need be only a set of lemmas provable from such an axiomatization. Thus, in verifying that a program maintains the loopfreeness of a list structure, we use as a basis a set of simple lemmas about the predicate Reach $(\mathrm{D}, \mathrm{X}, \mathrm{Y})$ instead of the definition in Section 2.1.4. These lemmas contain only universal quantifiers (often called quantifier-free lemmas) and are provable from the definition by induction. Attempting a verification directly from the definition would require a more powerful automated prover/simplifier than we currently have. However, in many examples, quantifierfree lemmas provide a natural way of defining concepts for specifying programs and are also sufficient to verify correctness.

Lemmas are stated in simple logical forms called replacement rules and inference rules. They contain information about how they are to be used in proof searches; the search methods are described in $[18,19]$, but are not of concern in this paper. A lemma of the form REPLACE $A$ BY $B$ is the equality $A=B$ or the logical equivalence $A \equiv B$, and INFER A FROM $B$ is the implication $B \Rightarrow A$.

The following two examples deal with verifying some properties of programs that manipulate lists. The first is insertion into a list; the second is an event counter queue implemented by a linear list. The examples show (a) the use of auxiliary predicates to express concepts such as loopfreeness of lists, (b) the characterization of concepts by lemmas in the basis, (c) documentation of the 
programs for verification, and (d) analysis of unproven VCs to locate possible bugs.

\subsection{Reachability in Linear Lists}

We wish to verify the loopfreeness of linear lists, in which each cell is a record with one pointer field, the Next field, which points to the next cell in the list. One way to approach this problem is to introduce a predicate $\operatorname{Reach}(\mathrm{D}, \mathrm{X}, \mathrm{Y})$, where $\mathrm{D}$ is a reference class term of type reference class of $T$, and $\mathrm{X}, \mathrm{Y}$ are both pointer variables of type $\uparrow T$. Reach $(D, X, Y)$ means that the sequence of pointers $X$, $D \subset X \supset . N e x t, D \subset D \subset X \supset . N e x t \supset . N e x t, \ldots$ in the reference class $D$ contains (or reaches) $Y$. This implies that the list structure between $X$ and $Y$ in $D$ is loopfree under the Next operation. Notice that Next ought to be an explicit parameter of Reach, but since we are assuming that our list structures have only one pointer field, we can omit it.

Example 2 is the insertion of an element into the middle of a linear list. We verify that Reach(D, Root, Sentinel) is still preserved after the insertion, Root and Sentinel being pointers to the beginning and end of the list.

Example 2.

type Ref $=\uparrow W o r d$;

Word = record Count: integer; Next: Ref end;

procedure Insert(Y, Root, Sentinel: Ref);

global (var P\#Word);

entry: $(\mathrm{Y} \neq$ Sentinel $) \wedge$ Reach $(\mathrm{P} \#$ Word, Root, $\mathrm{Y}) \wedge$ Reach $(\mathrm{P} \#$ Word, $\mathrm{Y}$, Sentinel $)$

$\wedge$ PointerTo(Root, P\#Word) $\wedge$ PointerTo(Sentinel, P\#Word);

exit: Reach (P\#Word, Root, Sentinel);

var Z: Ref;

Begin

NEW(Z);

$\mathrm{Z} \uparrow$. Next $:=\mathrm{Y} \uparrow$. Next;

$\mathrm{Y} \uparrow$. Next $:=\mathrm{Z}$;

end.

The set of lemmas below is a basis for verifying example 2. We do not claim that it is a complete axiomatization of Reach $(\mathrm{D}, \mathrm{X}, \mathrm{Y})$, but merely that each of the lemmas is an obvious property of Reach that can be deduced from the definition given in Section 2.1.4 by induction.

In the lemmas for Reach we introduce other predicates $\operatorname{InBetween}(\mathrm{D}, \mathrm{X}, \mathrm{Y}, \mathrm{Z})$ and NotInBetween $(D, X, Y, Z)$. The meaning of InBetween $(D, X, Y, Z)$ is "starting from $Y$ by taking Next successively one can reach $Z$ and one arrives at $X$ before arriving at Z." Therefore, $Z$ must be distinct from $Y$. NotInBetween(D, X, Y, Z) is the negation of InBetween $(D, X, Y, Z)$.

$\mathrm{G} 1$ states that for $\mathrm{W}$ to be reachable from $\mathrm{X}$ in a reference class resulting from class $D$ by performing $Y \uparrow . N e x t:=Z$ it is sufficient that $\operatorname{Reach}(D, X, Y)$ and $\operatorname{Reach}(\mathrm{D}, \mathrm{Z}, \mathrm{W})$ and also NotInBetween$(\mathrm{D}, \mathrm{Y}, \mathrm{Z}, \mathrm{W})$ to ensure that by the assignment operation the path from $\mathrm{Z}$ to $\mathrm{W}$ is not disconnected. Clearly the truth of this lemma depends on more atomic properties, e.g., Reach(D, Y, Y $\uparrow . N e x t)$, transitivity, and G2.

G3 states that changes to the list structure outside the segment between $\mathrm{X}$ and $\mathrm{Y}$ will not alter the reachability of $\mathrm{Y}$ from $\mathrm{X}$. 
G5 states that the newly created cell is not in between anything.

G6 states a sufficient condition for NotInBetween $(D, Y, Z, W)$ to be true.

\section{RULEFILE}

G1: Var D, W, X, Y, Z: INFER Reach( (D, CY D.Next, Z , X, W) FROM $\operatorname{Reach}(\mathrm{D}, \mathrm{X}, \mathrm{Y}) \wedge \operatorname{NotInBetween}(\mathrm{D}, \mathrm{Y}, \mathrm{Z}, \mathrm{W}) \wedge \operatorname{Reach}(\mathrm{D}, \mathrm{Z}, \mathrm{W})$;

G2: VAR D, X: INFER Reach(D, X, X) FROM X $\neq$ NIL;

G3: VAR D, E, X, Y, Z: INFER Reach((D, CZつ.Next, E), X, Y) FROM Reach(D, X, Y) $\wedge$ NotInBetween $(D, Z, X, Y)$.

G4: VAR D, X, R, S: INFER Reach $(D \cup\{X\}, R, S)$ FROM Reach(D, R, S) $\wedge R \neq X \wedge S \neq X$

G5: VAR D, X, Y, Z: REPLACE NotInBetween(D $\cup\{Z\}, Z, X, Y)$ BY TRUE;

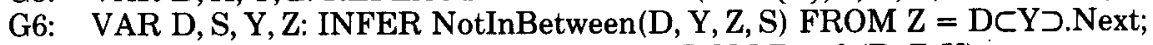

G7: VAR D, X, Y, Z: INFER Reach(D, X, Y) FROM Reach(D, Z, Y) $\wedge \mathrm{X}=\mathrm{D} \subset \mathrm{Z} \supset$.Next $\wedge \mathrm{Z} \neq \mathrm{Y}$

The verifier produces the following verification condition from example 2 and then the simplifier proves it using both the axioms for data structure terms (Section 2.1.3) and the rulefile (which is said to be the basis for this verification).

VERIFICATION CONDITION FOR THE MAIN PROGRAM IS:

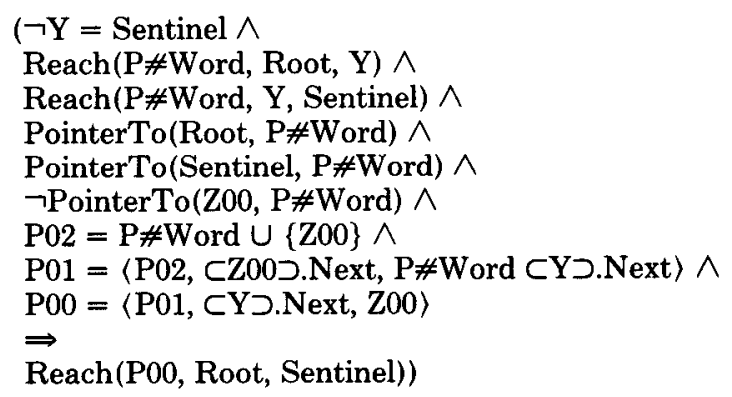

Example 3 illustrates what happens when we reverse the order of instructions in example 2. The program is no longer correct in that it does introduce a loop into a loopfree structure.

Example 3.

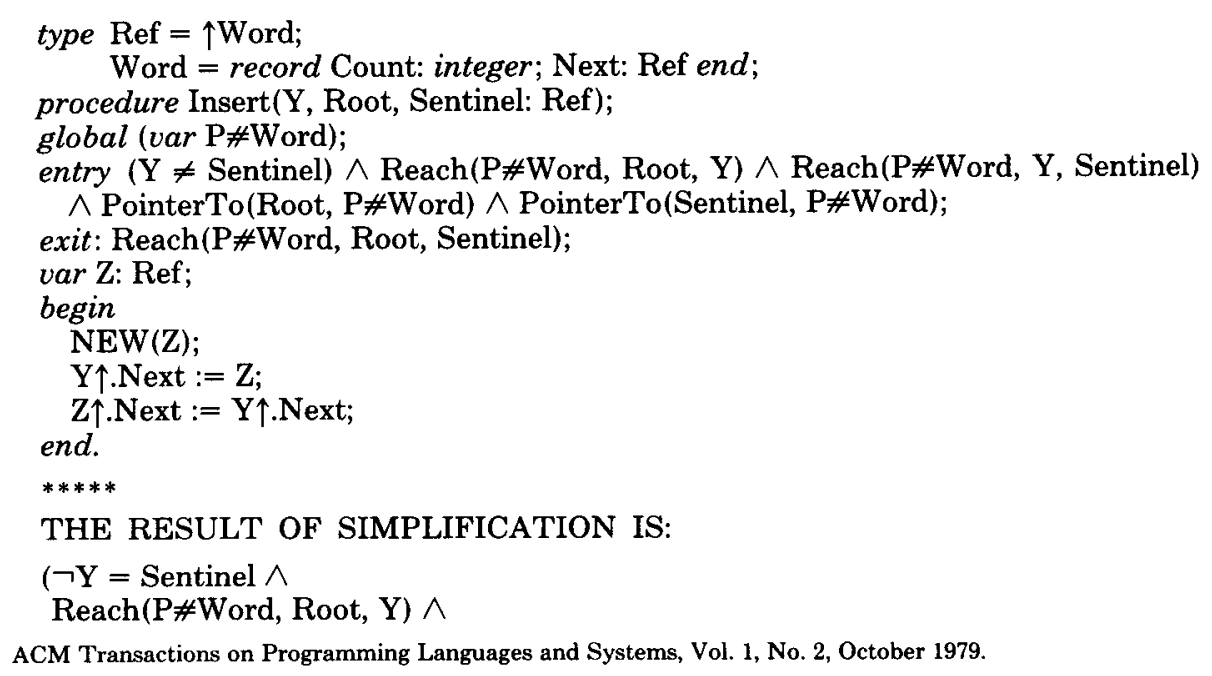




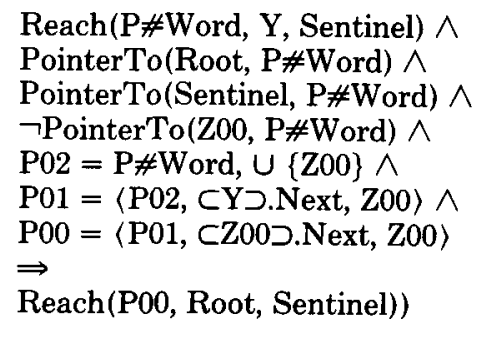

The result is obtained using the same rulefile as for example 2. The introduction of a loop into the list structure is obvious from the reference class expression (P01, СZ00つ.Next, Z00) for P00, the final state of the reference class. This term results from simplification using the data structure axioms. The second and third statements of the (bad) procedure are shown to imply $\mathrm{Z} \uparrow . \mathrm{Next}=\mathrm{Z}$ which clearly introduces a loop.

\subsection{Event Counter Queue Example}

This program was given to us by $\mathrm{N}$. Wirth as a challenge for verification. It manipulates a linear list to implement an event queue. Each cell of the list has three fields: Key, Count, and Next. The Key field contains the identification name for the cell, the Count field contains the number of times the Search is called with the corresponding Key, and the Next field contains the pointer to the next cell in the list. Root points to the first cell and Sentinel points to the next to the last cell. The last cell is a dummy cell.

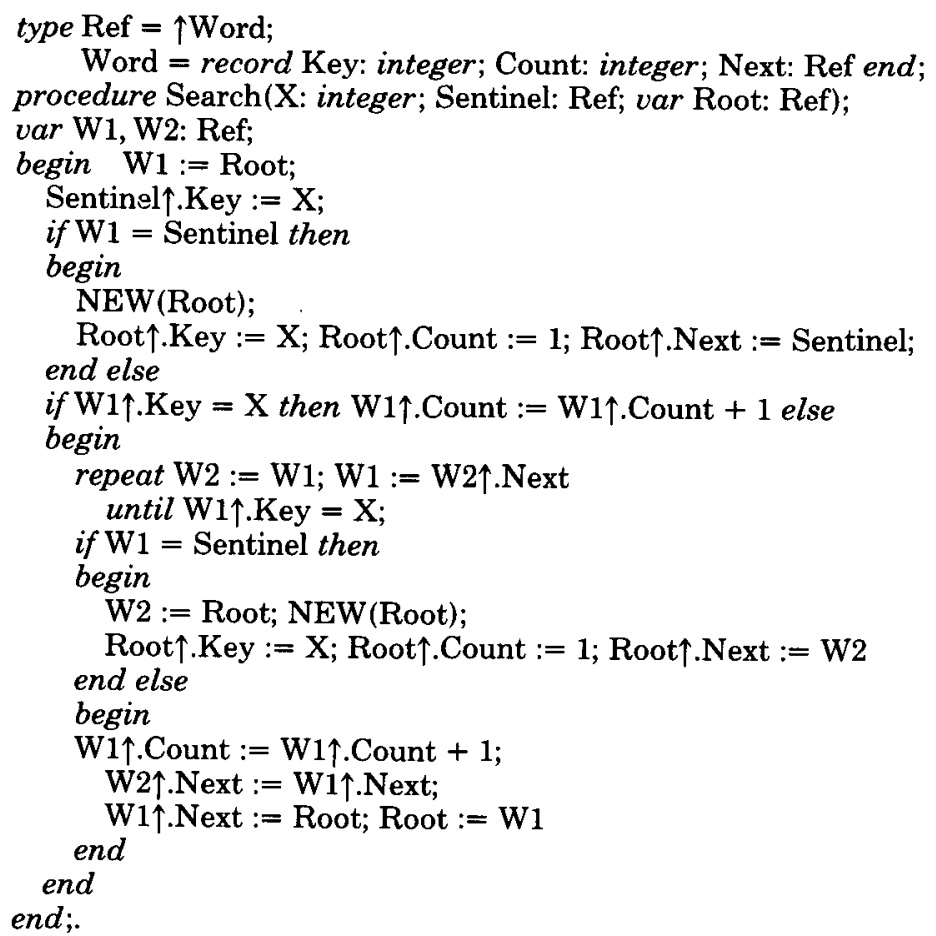


In order to verify this program we have to show that several properties hold. Here are some of them. (1) The list structure is always loopfree and Sentinel is reachable from Root. (2) If a cell with the given Key exists in the list, no new cell is added; otherwise, one cell is added. (3) After execution the list is reordered so that the first cell has the same Key as the given Key argument of Search, and the order of the other cells is unchanged. (4) Only the Count field of the cell with the given Key is incremented by 1 , and the rest are unchanged. (5) The program terminates. Here we are going to discuss a verification that the reachability property is maintained by Search.

Example 4 is the program with assertions about reachability. The entry and exit assertions state that loopfreeness is maintained. The only additional documentation is an invariant describing obvious properties of the variables in the repeat loop.

Example 4.

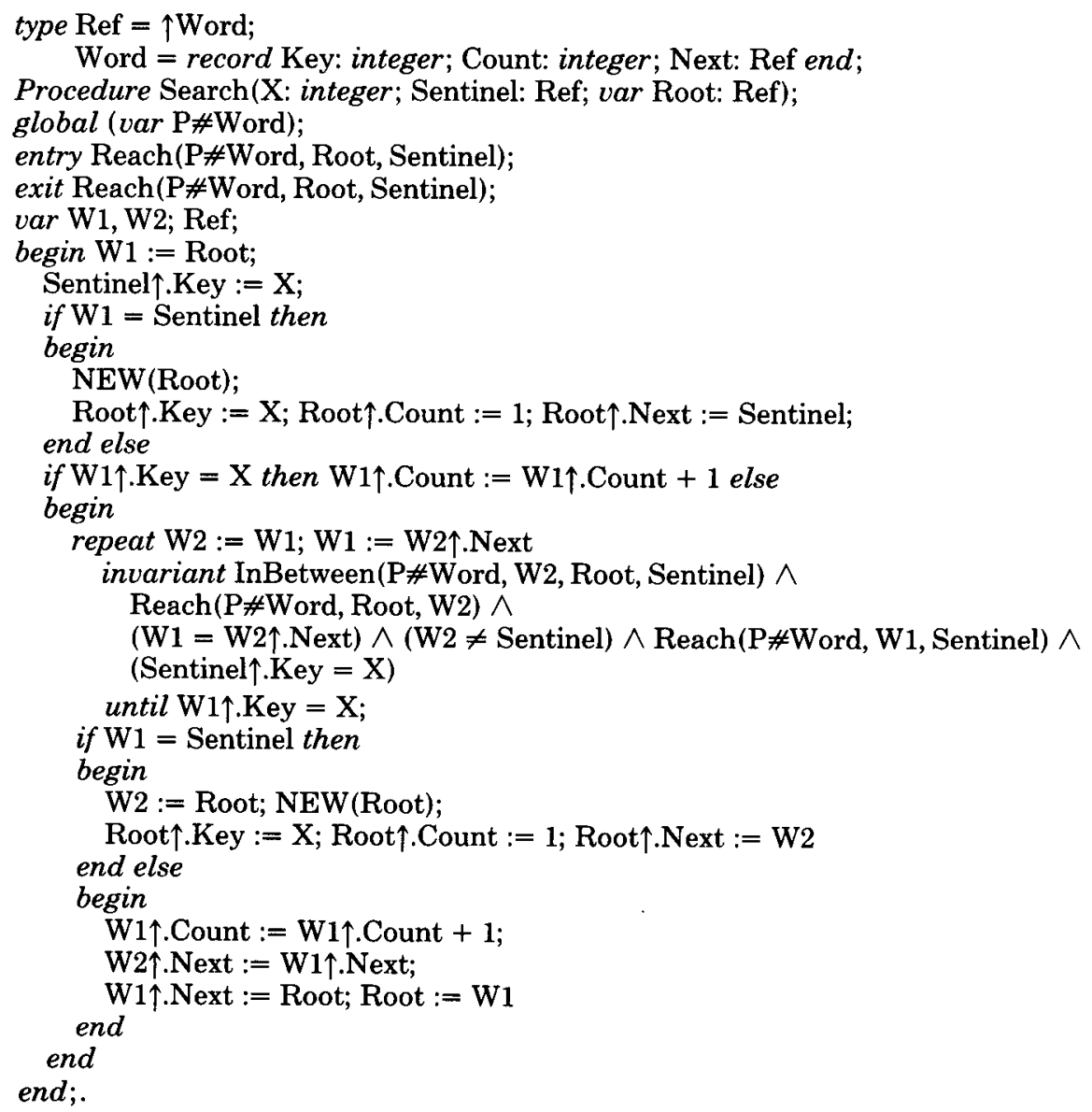

Example 4 may be verified using the lemmas for Reach given in the appendix together with the data structure axioms (Section 2.1.3). The rulefile in the appendix is in fact sufficient to verify that many simple list manipulating programs maintain loopfreeness. 


\section{CONCLUSION}

The axiomatic semantics for complex data structures was first implemented in the Stanford Pascal verifier in 1975 [18]. The introduction of reference classes in assertions resulted in the ability to specify Pascal pointer manipulations by assertions very similar to those used for array operations; the differences are that assertions about reference classes may contain the extension function and the PointerTo predicate. Other examples of specification and verification of pointer programs may be found in the references: [18] contains a study of the SchorrWaite algorithm, and [4] shows the verification of a benchmark routine for testing commercial Pascal compilers. The main problems encountered in specifying pointer programs that are not usually found with array programs result from the use of pointers to implement complicated structures (e.g., balanced binary trees). Specifications require the use of high level concepts describing properties of structures that do not have a standard axiomatization. So verification of pointer programs quite often depends on finding an elegant set of specification concepts and their axioms. Of course, the need to formalize high level concepts can also occur in verifying array programs (e.g., see the recent studies of verification decidability of Presburger array programs [20] and of an Insitu Permutation program [16]).

The Stanford Pascal verifier has gone through several development stages and is currently used for experiments at several research laboratories. It automates much of the work involved in analyzing a Pascal program for consistency with its specifications; to date, the largest programs shown to be consistent with rigorous logical specifications using this verifier are parts of a compiler, including a parser consisting of about 10 pages of Pascal. When such a system is available, the creative part of verification is the choice of specification concepts and their axioms. The tedious details of constructing consistency proofs are left to the verifier. Clearly there is a need for more research on program specifications, and the use of reference classes is just a beginning.

Following our use of reference classes as a specification technique for Pascal, the designers of Euclid went a step further and introduced the Collection type into the programming language. The axioms for Collections (8.1-8.8 of [11]) are given in a set theoretic formulation but are clearly equivalent to our axioms for reference classes except (i) 8.2 is an explicit assertion that a Collection is an unbounded set of values (we use axiom 14 to deduce the same thing). (ii) We do not axiomatize a FREE operation (8.8 of [11]) since deallocation of memory was not a Pascal operation.

Finally the DoD ADA language designers, who were aware of Euclid but not of our earlier work on Pascal verification, have reverted to treating the Collection (or reference class) as an implicit global parameter. We quote from the ADA design rationale [7]:

\subsubsection{Collections of Dynamic Variables}

Conceptually is it important to realize that each access type declaration implicitly defines a collection of potential dynamic variables. The actual collection will be built during program execution as allocators are executed. Its lifetime cannot be longer than that of the program unit in which the access type definition is provided.

Collections in the Green language are implicit and cannot be named (unlike those in early Pascal, Lis, and Euclid). The collections associated with different access types are 
always disjoint, i.e., two access variables of different access types are guaranteed to contain the internal names of dynamic records in different collections.

Finally, the collection associated with a given access type must be considered as part of the global environment that is accessible in the scope of the access type declaration.

[See our Section 2.2.2 on extensions to Pascal semantics.] It would seem that the use of reference classes as a specification concept for pointer operations may be here to stay.

\section{APPENDIX, RULEFILE FOR REACHABILITY}

\section{RULEFILE}

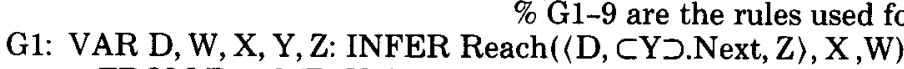
FROM Reach $(D, X, Y) \wedge \operatorname{NotInBetween}(D, Y, Z, W) \wedge \operatorname{Reach}(D, Z, W)$;

G2: VAR D, X: INFER Reach $(D, X, X)$ FROM X $\neq$ NIL;

G3: VAR D, E, X, Y, Z: INFER Reach( $(D, C Z \supset . N e x t, E\rangle, X, Y)$ FROM Reach(D, X, Y) $\wedge$ NotInBetween $(D, Z, X, Y)$.

G4. VAR D, X, R, S: INFER Reach(D $\cup\{X\}, R, S)$ FROM Reach(D, R, S) $\wedge \mathrm{R} \neq \mathrm{X} \wedge$ $\mathrm{S} \neq \mathrm{X}$

G5. VAR D, X, Y, Z: INFER Reach(D, X, Y) FROM Reach(D, Z, Y) $\wedge$ X $=$ DCZつ.Next $\wedge \mathrm{Z} \neq \mathrm{Y}$

G6: VAR D, X, Y, Z: INFER Reach(D, X, Y) FROM Reach(D, X, Z) $\wedge \operatorname{Reach}(D, Z, Y)$; $\%$ transitivity \%

G7: VAR D, X, Y: INFER Reach(D, X, DCYつ.Next) FROM Reach(D, X, Y) $\wedge$ Y $\neq N I L$;

G8: VAR D, X, Y, Z: REPLACE Reach( (D, CX J.Key, E $\rangle, Y, Z)$ BY Reach(D, Y, Z); $\%$ Key field does not affect reachability \%

G9: VAR D, X, Y, Z: REPLACE Reach( (D, CX J.Count, E $\rangle, Y, Z)$ BY Reach(D, Y, Z); $\%$ Count field does not affect reachability \% $\%$ G10-12 are the rules used for InBetween \%

G10: VAR D, X, Y, Z: INFER InBetween(D, X, Y, Z)

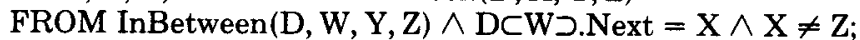

G11: VAR D, X, Y, Z: INFER InBetween(D, X, Y, Z) FROM X $=Y \wedge \operatorname{Reach}(D, Y, Z) \wedge Y$ $\neq \mathrm{Z}$;

G12: VAR D, W, X, Y, Z: REPLACE InBetween( $(D, C W \supset . C o u n t, E\rangle, X, Y, Z) \quad B Y$ InBetween $(D, X, Y, Z)$;

\% Count field does not affect InBetween \%

$\%$ G13-17 are the rules used for NotInBetween \%

G13: VAR D, X, Y, Z: REPLACE NotInBetween(D $\cup\{Z\}, Z, X, Y)$ BY TRUE;

G14: VAR D, S, Y, Z: INFER NotInBetween(D, Y, Z, S) FROM Z = DCY D.Next;

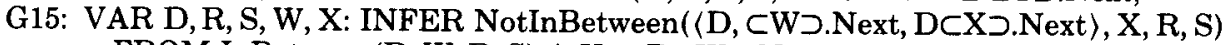
FROM InBetween(D, W, R, S) $\wedge \mathrm{X}=\mathrm{DCW}$.N.Next;

G16: VAR D, X, Y, Z: INFER NotInBetween(D, X, Y, Z) FROM Y = Z;

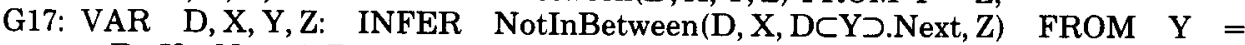

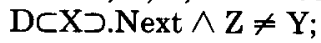

G18: VAR D, X, Y: INFER X $\neq$ NIL FROM Reach(D, X, Y);

$\%$ G18-20 are the rules about pointer inequality \%

G19: VAR D, X, Y, Z: INFER $X \neq N$ NIL FROM InBetween $(D, X, Y, Z)$;

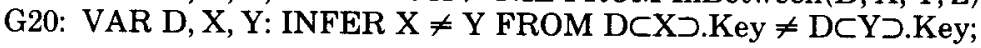

\section{ACKNOWLEDGMENT}

We wish to thank Jim Horning and the referees for helpful and detailed comments. We are grateful to $\mathrm{S}$. German for valuable comments.

\section{REFERENCES}

1. Burstall, R.M. Some techniques for proving correctness of programs which alter data structures. Machine Intell. 7 (Nov. 1972), 23-50. 
2. DeUtSCH, L.P. An interactive program verifier. Ph.D. Thesis, U. of California, Berkeley, 1973.

3. von Henke, F.W., AND Luckham, D.C. A methodology for verifying programs. Proc. Int. Conf. on Reliable Software. SIGPLAN Notices (June 1975), 156-164.

4. von HenKe, F.W., AND LUCKHAM, D.C. Verification as a programming tool. Stanford Verification Group Memo. Comptr. Sci. Dep., Stanford U. To appear.

5. HoARE, C.A.R. An axiomatic basis for computer programming. Comm. ACM 12, 10 (Oct. 1969), $576-580$.

6. HoARE, C.A.R., AND WIRTH, N. An axiomatic definition of the programming language PASCAL. Acta Informatica 2 (1973), 335-355.

7. Ichbiah, J.D., Krieg-Brueckner, B., Wichmann, B.A., Ledgard, H.F., Heliard, J-C., Abrial, J-R., Barnes, G.P., AND Roubine, O. Rationale for the design of the Green programming language. CII Honeywell Bull. (March 1979) (also SIGPLAN Notices, June 1979).

8. IGARASHI, S., LONDON, R.L., AND LUCKHAM, D.C. Automatic program verification I: logical basis and its implementation. Acta Informatica 4 (1975), 145-182.

9. KARP, R.A., AND LuCKHAM, D.C. Verification of fairness in an implementation of monitors. Proc. 2nd Int. Conf. on Software Engineering (Oct. 1976), 40-46.

10. KING, J.C. A program verifier. Ph.D. Thesis, Carnegie-Mellon U., 1969.

11. London, R.L., Guttag, J.V., Horning, J.J., Lampson, B.W., Mitchell, J.G., and Popek, , G.J. Proof rules for the programming language Euclid. Acta Informatica 10 (1978), 1-26.

12. LUCKHAM, D.C. Program verification and verification-oriented programming. Proc. IFIP Congress 77. North-Holland Publ. Co., Amsterdam, Aug. 1977, pp. 783-793.

13. Luckham, D.C., AND Suzuki, N. Verification of Array, Record, and Pointer operations in Pascal. Comptr. Sci. Dep. Rep., Stanford U. To appear.

14. McCARThy, J. Towards a mathematical science of computation. Proc. IFIP Congress 62 . NorthHolland Publ. Co., Amsterdam, 1962, pp. 21-28.

15. MoRris, J.H. Verification-oriented language design. Tech. Rep. 7, Dep. Comptr. Sci., U. of California, Berkeley, 1972.

16. PoLAK, W. An exercise in automatic program verification. To appear in IEEE Trans. Software Engineering.

17. Spitzen, J., and Wegreit, B. The verification and synthesis of data structures. Acta Informatica 4, 2 (1975), 127-144.

18. SuzukI, N. Automatic verification of programs with complex data structure. Ph.D. Thesis, Stanford U., 1976; reprinted in Outstanding Dissertations in the Computer Science. Garland Publ., Inc., 1979.

19. SuzukI, N. Verifying programs by algebraic and logical reduction. Proc. Int. Conf. on Reliable Software. SIGPLAN Notices (June 1975), 473-481.

20. SUzuri, N., AND JefFrerson, D. Verification decidability of Presburger array programs. To appear in $J . A C M$.

21. WIRTH, N. The programming language Pascal. Acta Informatica 1, 1 (1971), 35-63.

Received January 1976; revised September 1978 and June 1979 